\title{
(6) OPEN ACCESS \\ For a healthier future: a virtuous cycle for reducing exposure to persistent organic pollutants
}

\author{
Chisato Mori, ${ }^{1,2}$ Emiko Todaka ${ }^{2}$
}

'Department of

Bioenvironmental Medicine, Graduate School of Medicine, Chiba University, Chiba City, Japan

${ }^{2}$ Centre for Preventive Medical Sciences, Chiba University, Chiba City, Japan

Correspondence to Dr Chisato Mori, Department of Bioenvironmental Medicine, Graduate School of Medicine, Chiba University, 1-8-1 Inohana, Chuo-ku, Chiba City 260-8769 Japan; cmori@faculty.chiba-u.jp

Received 23 January 2017 Revised 19 April 2017 Accepted 24 April 2017 Published Online First 17 May 2017
CrossMark

To cite: Mori C, Todaka E. J Epidemiol Community Health 2017;71:660-662.

\section{ABSTRACT}

In the modern society, people are exposed to various pollutants during their lifetime. Worldwide, the status of children's health has changed in recent decades. Some studies have attempted to identify the causes of these changes and whether they relate to pollutant exposure; however, such attempts have faced major challenges because human life is complex, involving many social and environmental factors. Several long-term cohort studies are being conducted to determine the relationship between diseases and social and environmental factors in children. Even before we establish complete proof of adverse effects, we should attempt to decrease risk to future generations by adopting precautionary principles. Environmental exposure to persistent organic pollutants can be reduced throughout the stages of life- - the fetal period, newborn and infant periods, childhood, adolescence and adulthood (preconception) by individuals as well as by society as a whole. Through reducing environmental exposure to pollutants, adverse health effects can also be reduced, which will contribute to healthier future generations. Here, we suggest a virtuous cycle for improving the health of future generations through reduced exposure to persistent pollutants.

In the modern society, people are exposed to various persistent organic pollutants (POPs) during their lives, starting before conception and extending through fetal development, infancy, childhood, and adolescence to adulthood. According to many reports, humans are contaminated by POPs throughout their early lives. ${ }^{1}{ }^{2}$ In Japan, it was revealed that a group of infants younger than 2 years old who were breast fed had the same levels of polychlorinated biphenyls (PCBs) as people over 60 years old $^{3}$ (figure 1 ). PCBs are a representative POP; if an individual has relatively high levels of PCBs, they are more likely to have higher levels of other POPs. ${ }^{4}$

In general, the health of children worldwide has worsened according to various measures over the past few decades. Increasing numbers of children are obese, and obesity contributes to a higher risk of developing diabetes in later life. There are reports showing that the number of children who suffer from allergies (such as asthma, atopic dermatitis and rhinitis) or are diagnosed with neurodevelopmental disorders (such as autism spectrum disorders, including Asperger's syndrome, attention deficit hyperactivity disorder and learning disability) has been increasing over the past few decades. ${ }^{1}$ Recent reports have shown an association between childhood POP exposure and prevalence of neurodevelopmental disorders. ${ }^{5}{ }^{6}$ Furthermore, environmental factors in early life can affect the development of diseases with later onset, such as diabetes and hypertension. This phenomenon is termed the $\mathrm{DOHaD}$ (developmental origins of health and disease) and has been studied closely. ${ }^{78}$

Studies have attempted to identify the causes of the above changes in children's health; however, such attempts have faced major challenges because human life is complex, involving many social and environmental factors. A long-term cohort study involving children is one of the few ways by which it is possible to ascertain the causes of changes to children's health. Several such cohort studies are being conducted to determine the relationship between diseases (or symptoms) and social and environmental factors. ${ }^{9}$ Some such associations have already been identified. For example, one recent long-term cohort study determined that maternal smoking during pregnancy was associated with low birth weight of neonates. ${ }^{10}$ Another such study found that maternal PCB exposure was related to low birth weight in neonates. ${ }^{11}$

Therefore, preventive measures to reduce environmental pollution exposure before pregnancy are necessary to reduce disease and health-related risks for future generations. We identified three measures that could decrease these risks if implemented proactively.

The first measure is to reduce the level of POPs in the immediate environment through advocacy, education, administrative measures (such as developing guidelines) and political approaches.

The second measure is to prevent POP exposure that occurs through eating, drinking, breathing in and touching contaminated substances. For example, if people of reproductive age avoided eating large fish, fatty meat, and dairy products that have relatively high levels of POPs, their exposure levels would decrease before conception. Cooking methods can also affect levels of POP exposure. Boiling or grilling meat and fish can reduce the level of POP contamination. ${ }^{12}$ For newborns, breast milk is clearly the ideal source of nutrition. Breast feeding has numerous benefits, such as fostering attachment between mother and newborn and conferring immunity from mother to child. However, breast milk in women from developed countries contains high levels of fat-soluble contaminants and exposure to these contaminants has been reported to have health risks for infants. ${ }^{3} 6$ Thus, in areas where clean water and alternative foods are available, shortening the breastfeeding period to 1 year at maximum may reduce risk. ${ }^{3}$

The third measure is to reduce the level of internal exposure to POPs by eating fibrous foods. Green vegetables, such as spinach and kale, and 


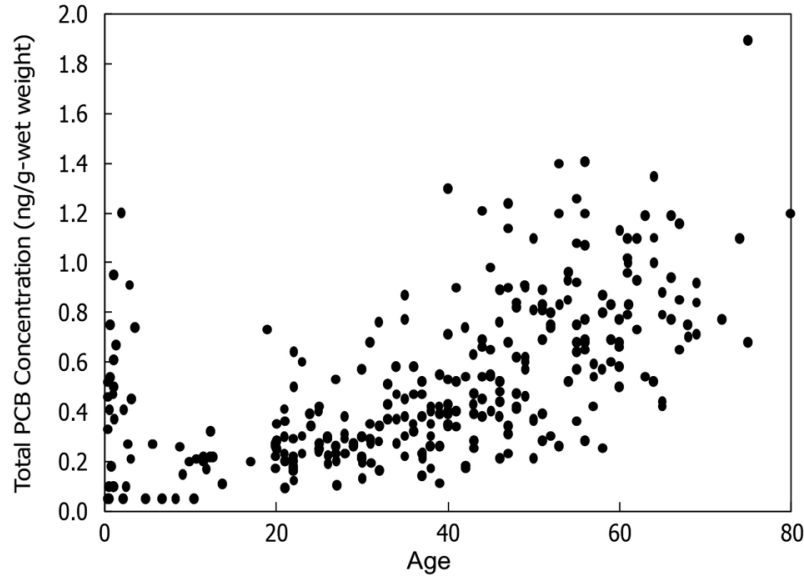

Figure 1 Blood PCB levels in a Japanese population. The blood PCB levels of 580 Japanese individuals aged $0-80$ years. A group of highly contaminated individuals under 2 years old should be noted. PCB, polychlorinated biphenyl.

chlorophyll from chlorella supplements can decrease contaminants in the human body. ${ }^{13}$ In cases of abnormally high contamination with PCBs or dioxins, such as following an accident, a medication for hyperlipoproteinaemia-colestimide (anion-exchange resin)—can be used to reduce the contamination level. ${ }^{14}$

If the above measures are implemented, a virtuous cycle of reducing environmental exposure and producing healthier future generations will become possible (figure 2).

In the modern society, people accumulate POPs through daily exposure (here, the 'contaminated adult' stage). If individuals do not adopt measures to reduce their level of contamination, then those contaminants can be transferred from parents to fetuses. If people of reproductive age reduce the contaminants in their body before conception occurs, they can decrease pollutant levels in sperm and oocytes. If expecting mothers reduce their intake of fatty meat and fatty fish during pregnancy, they could reduce their exposure to environmental chemicals. Lower levels of exposure to the mother would then lead to diminished transplacental exposure and a less contaminated uterine environment (the 'fetal stage').

As noted above, reducing the breastfeeding period to a maximum of 1 year has been shown to decrease POP levels for newborns and infants. ${ }^{3}$ However, if the level of contaminants in the mother's body can be reduced before pregnancy so that breast milk is less contaminated, the breastfeeding period should not necessarily be shortened (ie, during the 'newborn and infant stage').

Childhood is a unique period, during which children's bodies develop rapidly and they are exposed to contaminants in a manner that is different from that of adults. For example, children are nearer to the ground than adults, and so children are more exposed to contaminants from soil and the like. Children are also more likely to be exposed through habits like eating soil and things that they find on the ground or floor. In addition, children's respiratory volume per kilogram of body weight is much greater than that of adults; thus, children's exposure to air pollutants may be higher. If children could decrease the POPs they absorb through eating, drinking and touching, it would result in less contamination in adolescence. ${ }^{1}$ For people of reproductive age (from adolescence through adulthood), it is beneficial to avoid contaminated food and to choose an

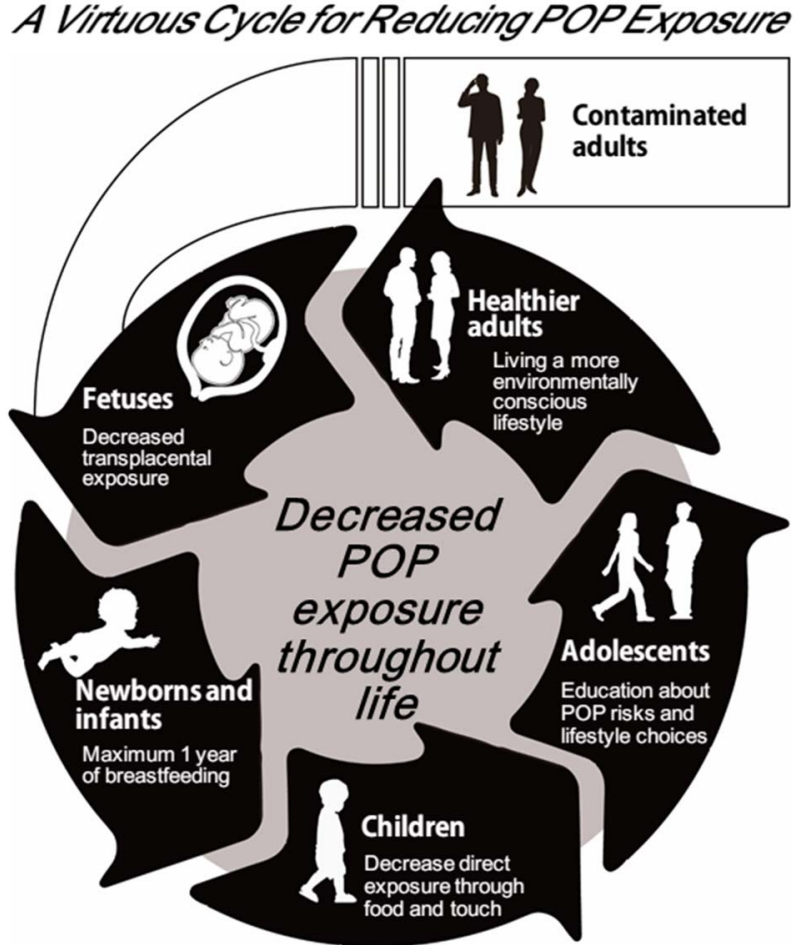

Figure 2 A virtuous cycle for reducing environmental persistent organic pollutant (POP) exposure. It is highly important to improve the safety of the environment by reducing the risk of persistent pollutant contamination through administrative and political measures. However, individuals can also take steps to reduce their internal and external exposure to persistent contaminants in the environment. There are five main developmental periods in life, and each period demands a different approach for decreasing exposure.In the modern society, people accumulate POPs in their bodies (ie, the 'contaminated adults' stage); this contamination may be associated with health risks. If people of reproductive age reduced the persistent pollutant levels in their bodies before conception, they could decrease contamination in sperm and oocytes.

1. Fetal period: During pregnancy, if mothers reduced their intake of fatty meat and fatty fish, they would decrease their exposure to persistent environmental contaminants. This reduced exposure could lead to diminished transplacental exposure and a less contaminated uterine environment.

2. Newborns and infants: Reducing the breastfeeding period to a maximum of 1 year could decrease POP contaminant levels in newborns and infants.

3. Children: Childhood is a unique period during which children's bodies develop rapidly and they become exposed to contaminants in a manner that is different from that of adults. If children could decrease the contaminants that they absorb through eating, drinking and touching objects, they would have lower contamination as adolescents.

4. Adolescents: Adolescence is an important period for people to become healthier adults. Advocacy and education on environmental health can play an important role during this period.

5. Healthier adults: If adults are educated about the health risks of environmental contaminants, they are likely to adopt an environmentally conscious lifestyle. Such a lifestyle would, in turn, reduce the pollutants to which individuals are exposed through good eating habits and avoiding contaminants. This decreased exposure would then result in a better uterine environment for fetuses.

If this cycle were implemented in its entirety, it could result in healthier future generations. 
environmentally conscious lifestyle to set the stage for a healthier next generation. ${ }^{15}$

Adolescence is an important period for people to become healthier adults. Like sex education, health advocacy and education related to environmental risks can play an important role in establishing health habits. If children learn about environmental contaminants, safer foods and drinks, and better ways of living to avoid exposure to pollutants, they will make better choices, which will result in lower contamination levels among adults.

Adults who are well educated regarding environmental health risks are likely to adopt an environmentally conscious lifestyle, and reduce the persistent pollutants to which they are exposed through good eating habits and avoiding contaminants. This reduced risk of contamination would then result in a better uterine environment for fetuses. Such practices would complete the virtuous cycle of reducing exposure to persistent environmental pollutants. This cycle would lead to less contaminated, healthier future generations.

Finally, to protect future generations from environmental contaminants, advocacy for young people would be absolutely essential. It is particularly important to increase the number of health professionals, such as paediatricians, obstetricians, gynaecologists, nurses and others involved in the health sector, who are interested in this subject and possess the appropriate scientific knowledge. Adding environmental health as part of the curriculum in medical education courses could also be an effective way to increase the number of young people who are interested in this topic.

The cycle we suggest here is limited to POP exposure and the suspected health risks from exposure to POPs, which are already known. However, if the data obtained from longterm cohort studies in children were analysed by omics analysis of individuals' exposome, epigenome, microbiome and metabolome, it may be possible to identify relationships between unexpected environmental contaminants and health risks. ${ }^{16}$

It is suspected that the mechanisms of $\mathrm{DOHaD}$ are triggered by epigenetic alterations caused by environmental chemicals. By analysing omics data from cohort studies, it is possible to determine what kind of epigenetic alterations are caused by which environmental chemicals ${ }^{17}$ and what diseases these alterations might trigger. $^{8}$ Furthermore, some reports insist that environmental exposure to chemicals can change the microbiome, ${ }^{18}$ leading to diseases and conditions such as allergies among mothers or children. ${ }^{19}$

Theo Colborn, one of the authors of Our Stolen Future, ${ }^{20}$ argues that the same budget should be apportioned to studying the inner space of the human body as that for investigating outer space. For the modern society, protecting future generations from environmental pollutants represents a major challenge because such pollutants are being produced on an ever greater scale. However, it should be the mission of the current generation to decrease environmental pollutant exposure and create a better living environment for all.

\section{What is already known on this subject}

- More attention should be paid to the fact that babies are born contaminated and more measurements to reduce the contamination level should be encouraged to develop.

\section{What does this study add}

Broader virtuous cycle will contribute to a cleaner human body and save future generations.

Contributors CM planned the essay. Both authors contributed for the discussion. ET wrote the draft and revised. Both authors reviewed, edited and agreed to publish this essay. CM is responsible for the overall content as guarantor.

Funding This work was supported by MEXT KAKENHI, grant number JP16H01781. Competing interests None declared.

Provenance and peer review Commissioned; externally peer reviewed.

Open Access This is an Open Access article distributed in accordance with the Creative Commons Attribution Non Commercial (CC BY-NC 4.0) license, which permits others to distribute, remix, adapt, build upon this work non-commercially, and license their derivative works on different terms, provided the original work is properly cited and the use is non-commercial. See: http://creativecommons.org/ licenses/by-nc/4.0/

\section{REFERENCES}

1 Mori C, Todaka E. Environmental contaminants and children's health; Sustainable health science for future generations. Tokyo, Japan: MARUZEN PLANET Co.,Ltd., 2011; (ISBN: 978-4-86345-077-6). http://cpms.chiba-u.jp/enquete/consent.php (15 January 2017).

2 Fukata $\mathrm{H}$, Omori $\mathrm{M}$, Osada $\mathrm{H}$, et al. Necessity to measure PCBs and organochlorine pesticide concentrations in human umbilical cords for fetal exposure assessment. Environ Health Perspect 2005;113:297-303.

3 Mori C, Kakuta K, Matsuno Y, et al. Polychlorinated biphenyls levels in the blood of Japanese individuals ranging from infants to over 80 years of age. Environ Sci Pollut Res 2014:21:6434-9.

4 Ochiai S, Shimojo N, Yuka I, et al. A pilot study for foetal exposure to multiple persistent organic pollutants and the development of infant atopic dermatitis in modern Japanese society. Chemosphere 2014;94:48-52.

5 Lee DH, Jacobs DR, Porta M. Association of serum concentrations of persistent organic pollutants with the prevalence of learning disability and attention deficit disorder. J Epidemiol Community Health 2007:61:591-6.

6 Solomon GM, Huddle AM. Low levels of persistent organic pollutants raise concerns for future generations. J Epidemiol Community Health 2002;56: 826-7.

7 Barker DJP, Martyn CN. The maternal and fetal origins of cardiovascular disease. J Epidemiol Community Health 1992;46:8-11.

8 Gluckman P, Hanson M. Developmental origins of health and disease. Cambridge, UK: Cambridge University Press, 2006.

9 Kawamoto T, Nitta H, Murata K, et al. Rationale and study design of the Japan environment and children's study (JECS). BMC Public Health 2014;14:25.

10 Suzuki K, Shinohara R, Sato $M$, et al. Association between maternal smoking during pregnancy and birth weight: an appropriately adjusted model from the Japan Environment and Children's Study. J Epidemiol 2016;26:371-7.

11 Eguchi A, Sakurai K, Watanabe M, et al. Exploration of potential biomarkers and related biological pathways for PCB exposure in maternal and cord serum: a pilot birth cohort study in Chiba, Japan. Environ Int 2017;102: 157-64.

12 Wilson ND, Shear NM, Paustenbach DJ, et al. The effect of cooking practices on the concentration of DDT and PCB compounds in the edible tissue of fish. J Expo Anal Environ Epidemiol 1998;8:423-40.

13 Morita K, Ogata M, Hasegawa T. Chlorophyll derived from Chlorella inhibits dioxin absorption from the gastrointestinal tract and accelerates dioxin excretion in rats. Environ Health Perspect 2001;109:289-94.

14 Sakurai K, Todaka E, Saito Y, et al. Pilot study to reduce dioxins in the human body. Intern Med 2004:43:792-5.

15 Mori C, Todaka E. Establishment of sustainable health science for future generations: from a hundred years ago to a hundred years in the future. Environ Health Prev Med 2009;14:1-6.

16 Sakurai K, Miyaso H, Eguchi A, et al. Chiba study of Mother and Children's Health (C-MACH): cohort study with omics analyses. BMJ Open 2016:6:e010531.

17 Stein RA. Epigenetics and environmental exposures. J Epidemiol Community Health 2012;66:8-13.

18 Choi JJ, Eum SY, Rampersaud E, et al. Exercise attenuates PCB-induced changes in the mouse gut microbiome. Environ Health Perspect 2013;121:725-30.

19 Riiser $\mathrm{A}$. The human microbiome, asthma, and allergy. Allergy Asthma Clin Immunol 2015:11:35.

20 Colborn T, Dumanoski D, Myers JP. Our stolen future. USA: Dutton, 1996 (ISBN, 978-3-16-148410-0). 